\title{
¿Lo racional del ser humano es una entelequia? Las emociones y el aprendizaje
}

\author{
Is the rational thought of humanity an entelechy? \\ Emotions and learning
}

Olga Gonzalez Sarmiento ${ }^{1}$

\section{RESUMEN}

El presente artículo profundiza en la relación explorada por la neurociencia sobre el tema de las emociones y su relación con el aprendizaje humano. Se intenta dar una explicación sobre aquellos procesos que tienen lugar en el cerebro, específicamente, en el sistema límbico donde se generan todo tipo de emociones, tanto positivas como negativas que intervienen, sin a veces, ser tenidas en cuenta, de manera importante en el proceso educativo. Asimismo, se proponen algunos cambios y estrategias pequeños, pero significativos, que pueden coadyuvar al logro de mejores aprendizajes en nuestros estudiantes.

\section{Palabras clave}

Aprendizaje Humano, Emociones, Sistema Límbico, Neurociencia.

\section{ABSTRACT}

This paper studied in depth the relationship that had been explored by the Neuroscience between the emotions and human learning. It was an attempt to explain such processes originated specially on the limbic system, where all kind of positive and negative emotions are generated, without being sometimes taken into account in the educational process in a significant way. Moreover, some small but important, changes and strategies are proposed to contribute to the achievement of better learning in our students.

\section{Keywords}

Human learning, Emotions, Limbic system, Neuroscience.

Nuestro cerebro es un órgano hecho para modificarse en respuesta a las experiencias Richard Davidson, 2010

Muchas veces hemos oído definir nuestra especie como "seres racionales", sin embargo, según investigadores como Daniel Kahneman o Gerd Gigerenzer, la racionalidad no vendría a ser, precisamente, la forma básica en que acostumbramos actuar los seres humanos.
Kahneman, catedrático de psicología de la Universidad de Princeton, ha profundizado en la toma de decisiones en situaciones asociadas al riesgo y la incertidumbre. Para este investigador, quien obtuvo un Premio Nobel en Economía en el 2002, sin ser economista,

1 La Dra. Olga González Sarmiento se formó en la Universidad Femenina del Sagrado Corazón y en la Pontificia Universidad Católica del Perú, actualmente centra sus intereses en temas asociados a la educación superior y al campo de la psicología clínica. 
la definición de racionalidad: Actitud del que actúa de acuerdo con la razón y no se deja llevar por sus impulsos, no podría ser, en la mayoría de los casos, lo que nos defina.

Según Kahneman, cuando pensamos, podemos optar por dos vías bastante disímiles entre sí. Un primer sistema se caracteriza por ser muy intuitivo y emocional y, por supuesto, muy rápido, al ritmo de la emoción que lidera en ese momento a la persona; un segundo sistema, coincidiría más con la definición que citamos previamente: un pensamiento analítico, reflexivo y, por supuesto, racional.

Lo peculiar es que, según este estudioso de los comportamientos humanos, "no siempre reflexionamos sobre cuál de los dos sistemas ha tomado las riendas de nuestro comportamiento", el de las conclusiones automáticas o el de las respuestas conscientes, en un determinado momento de nuestra vida.

Por otro lado, Gerd Gigerenzer, psicólogo y director del Instituto Max Planck de Desarrollo Humano en Berlín que ha profundizado por años en el estudio de la toma de decisiones, el ser humano piensa en base a heurísticos que subyacen a las intuiciones. Es decir, pensamos en función a una serie de reglas generales que hemos aprendido a través de la evolución, por lo tanto, tendemos a desechar información que consideramos "superflua" y nos dejamos llevar por la intuición para escoger un empleo nuevo, una determinada dirección en una ciudad nueva o, inclusive, al momento de elegir una pareja. En una entrevista, menciona que "la intuición nos lleva a tomar decisiones más rápida y acertadamente, en la mayoría de los casos, que si racionalizamos demasiado".

Peter Gardenfors (2005) en su artículo "La intuición como conocimiento implícito" sostiene que las intuiciones humanas se basan, principalmente, "en conocimiento implícito que es difícil hacer explícito". Es decir, sabemos algo, sin muchas veces, poder explicarnos cómo lo sabemos. Por tanto, se trata de un conocimiento que no ha seguido un camino racional en el proceso de su construcción, sino un conocimiento nacido de una comprensión intuitiva que involucra, a su vez, una reacción repentina de tipo emotivo.

Cuando mencionamos a la emoción estamos adentrándonos en un campo que puede resultarnos bastante familiar y cotidiano; no ha sido gratuito el que los seres humanos hayamos sido catalogados como "seres en emoción".

Pero ¿a qué nos referimos cuando hablamos de "emoción"?

L. Carretié (2011) define a la emoción como "un fenómeno mental omnipresente" que puede manifestarse de diferentes formas. Una de ellas como la reacción puntual a un evento, (el inicio de un movimiento sísmico, por ejemplo) o como la asociación de eventos o contextos a experiencias placenteras o displacenteras que nos hayan quedado "grabadas" emocionalmente.

La experiencia nos ha enseñado que cuando los seres humanos nos emocionamos, pueden ocurrir una serie de variaciones en nuestro comportamiento habitual; todas ellas debido a que las emociones son fenómenos que poseen carácter biológico y cognitivo a la vez. Es así que podemos considerar a las emociones como reacciones complejas. Algunos autores consideran que pueden ser descritas en función a tres elementos que les conforman: un estado mental específico, un cambio fisiológico (generalmente asociado al estado mental que se está experimentando) y un impulso a actuar. Si volvemos al ejemplo del sismo que anteriormente citamos, la emoción que se genera es la de miedo (el estado mental).

Al experimentar miedo es probable que los músculos de nuestro cuerpo se tiemplen, las retinas de nuestros ojos se agranden, los latidos de nuestro corazón se aceleren y la respiración se vuelva superficial (cambios fisiológicos), y ya que nuestro cuerpo está preparado para el enfrentamiento o la huida, decidimos huir a través de la salida más cercana (impulso a actuar).

También por propia experiencia sabemos que existen diferentes tipos de emoción y, 
por supuesto, diferentes formas de responder frente a ellas. Por ejemplo, cuando la emoción está asociada a cualquier forma de placer, la reconocemos como positiva y nos sentimos felices o gozosos, es el caso cuando sentimos atracción por alguien y somos correspondidos. Todo lo contrario sucede cuando alguien abusa de nuestra confianza, o nos defrauda, puede que percibamos la situación-estímulo como una amenaza. Entonces, experimentamos, según sea el caso, tristeza, desilusión, cólera, etc.

Frente a tales situaciones, cada uno de nosotros va a responder de distinta manera, algunos lo harán de forma intensa; otros, no tanto. Lo que, al parecer, sí tenemos en común la mayoría de los seres humanos es que somos seres que estamos sujetos a la emoción a lo largo de nuestras vidas.

Goleman (1995), por otro lado, sostiene que "cuando estamos dominados por el anhelo y la furia, trastornados por el amor y retorcidos de temor, es allí cuando se puede decir que se está dominado por el cerebro límbico".

El término 'límbico' fue acuñado en el año 1878 por el médico y científico francés Paul Broca, para designar un área relacionada con el aprendizaje, la memoria y las respuestas emocionales. Dicha zona está situada justo debajo de la corteza cerebral y está formada por el tálamo, el hipotálamo, la amígdala cerebral y el hipocampo.

El "cerebro límbico", como lo denomina Goleman, o "sistema límbico" como lo denominan los neurocientíficos actuales, es un conjunto de estructuras del encéfalo, conformado por el cerebro, cerebelo y bulbo raquídeo, que están conectadas entre sí y cuya función tiene que ver con los estados emocionales de los seres humanos o con lo que denominamos "instintos". Es así como rige nuestras emociones más primitivas, es decir, aquellas relacionadas a la supervivencia o al comportamiento sexual asociado a la reproducción.

Asimismo, es necesario precisar que, existe en el sistema límbico una estructura que podría considerarse como la más importante; es la que se denomina amígdala cerebral. Esta región específica, con forma de una almendra, está compuesta por dos núcleos neuronales que se encuentran situados en la parte más profunda de los lóbulos temporales y se encarga de almacenar y manejar las emociones más primitivas del ser humano. Básicamente, podría decirse que la principal labor de la amígdala es la de integrar las emociones experimentadas por el sujeto, con aquellos patrones aprendidos durante toda su vida; provocando así cambios temporales en su fisiología o la preparación para emitir algún tipo de conducta en respuesta al estímulo registrado.

En este contexto, podría afirmarse que la función desempeñada por la amígdala presenta dos aspectos distintivos. El primero, la gestión de emociones que realiza nos permite responder a estímulos con acciones de defensa, ataque o huida, siempre con fines de supervivencia. En el segundo, la amígdala es la que se encarga de revivir aquellos aspectos traumáticos de nuestra infancia y nos prepara también para experimentar la ira y/o el miedo. Por lo antes dicho, la amígdala es reconocida como el principal núcleo de control de las emociones y sentimientos en el cerebro.

Los hallazgos realizados por la neurociencia en los últimos años nos permiten conocer un poco más sobre el proceso que sucede al interior del cerebro humano al generar una emoción. Todo se inicia cuando ingresa un estímulo a nuestros sentidos. La corteza cerebral informa a la amígdala y ella decide si el estímulo significa una amenaza o no, es decir, decide si debe responder enfrentándose (con agresividad) o huyendo (con miedo). Una vez que la amígdala ha escogido la emoción que ayudará a la supervivencia del sujeto, procede a enviar señales a otros lugares del cerebro, a fin de desencadenar la denominada emoción interna. Por otro lado, también desencadena la expresión de dicha emoción (emoción externa).

Pongamos un ejemplo: Un niño se encuentra pedaleando alegremente en su bicicleta cuando de pronto surge entre los matorrales de un jardín un perro grande que lo empieza 
a perseguir a toda carrera. En el interior del cerebro, lo que ha sucedido es que la corteza visual del niño ha enviado la imagen del animal a la amígdala, esta ha decidido que representa una amenaza, por lo que, a su vez, ha enviado una orden al hipotálamo para que ponga en marcha todo el sistema de emergencia frente a un peligro. La amígdala decide entonces que es una amenaza de la que hay que huir. El cerebro del niño experimenta miedo (emoción interna), y su corazón empieza a latir rápidamente, la respiración se agita y se vuelve superficial, los vellos de su cuerpo se erizan y los músculos se tornan tensos. Es entonces que el niño aprieta la marcha y huye. En un momento dado, se da cuenta de que el animal ya no lo persigue, y siente una emoción de alivio, todos los signos de alarma van desapareciendo y su cuerpo recupera la homeostasis, lo que le permite continuar tranquilamente con su paseo.

La situación descrita previamente coincide con lo dicho por Holodynski (2009) quien define a la emoción como una configuración de cambios fisiológicos periféricos que tienen lugar en el cuerpo, así como formas de expresión y sentimientos subjetivos que dependen de la evaluación de la situación emocional evocada. Aparte de lo dicho, también es necesario precisar que el circuito de recompensa del cerebro, en especial, lo que se encuentra asociado con el placer y las adicciones, tiene su punto de partida en el sistema límbico del cerebro.

Debe mencionarse, por otro lado, que lo límbico en el ser humano no involucra exclusivamente lo que respecta a la motivación de consumo de sustancias psicoactivas, sino también a aquello que tiene que ver con la conducta compulsiva y las recaídas que experimenta una persona adicta en su búsqueda del placer.

Asimismo, debe tenerse en cuenta que, aunque dicho sistema esté probadamente asociado a las emociones humanas, también los científicos han descubierto que resulta de vital importancia para la motivación, el desarrollo de aprendizajes y la memoria. Por tanto, el sistema límbico no solo está asociado al control de las emociones y la búsqueda de la supervivencia del individuo, también lo está a la motivación intrínseca, la iniciativa, los estados de alerta y la cognición.

Otro de los importantes hallazgos realizados por la neurociencia está relacionado con el aprendizaje humano. En este contexto, el aprender algo, el construir nuevos significados sobre hechos, experiencias y situaciones o el reflexionar sobre el propio mundo interior genera variaciones en las conexiones sinápticas existentes y con ellas cambios en nuestro cerebro, en otras palabras, un buen aprendizaje, literalmente, modifica nuestro cerebro.

En otras palabras, todo lo que constituya novedad enriquece nuestro cerebro, todo lo que signifique emoción, cambia nuestro cerebro. Visitar una exposición artística donde se utilicen técnicas desconocidas para nosotros, la lectura y profundización de un nuevo tema de interés, tener experiencias distintas a lo anteriormente vivido, desarrollar habilidades antes no exploradas (cantar, ensayar una danza, tocar un instrumento musical, aprender un nuevo idioma, etc.) resultan beneficiosas para nuestro cerebro.

Lo que debemos considerar en el estudio del aprendizaje humano asociado al estudio de los hallazgos de la neurociencia es que, gracias a nuestro interés por aprender, nuestro cerebro se adapta y readapta constantemente. Se ajusta a nuevas realidades, se enriquece cada vez que decidimos explorar nuevos campos de conocimiento; en pocas palabras, se estimula cuando aprendemos algo que le significa novedad o le emocione.

Los datos que han recogido los numerosos estudios realizados sobre el cerebro que aprende, nos indican que el proceso inicia con lo que mencionábamos previamente, con un estímulo, de preferencia, novedoso. Así, se ha comprobado que nuestro cerebro se activa, cuando aprendemos temas sobre los que poseemos cierto conocimiento ya existente, pero, que se activa mucho más cuando el aprendizaje se refiere a un tema inexplorado por nosotros previamente. 
Al respecto, Donald Hebb (1949) postuló en su Teoría de la asamblea celular, conocida como la Ley de Hebb, que el aprendizaje se produce cuando una célula requiere menos impulsos eléctricos de otra la siguiente vez que se activa. Sucede todo lo contrario cuando es la primera vez que se da una conexión entre ambas, en ese caso, la conexión es de alta intensidad; es decir, un aprendizaje nuevo, un estímulo novedoso trae consigo conexiones neuronales más fuertes.

Por lo antes dicho, podría afirmarse que la clave del aprendizaje radica en estar permanentemente aprendiendo algo nuevo, pues cuando lo hacemos, estamos cambiando la forma como nuestro cerebro se conecta, lo que ayuda a mantener su plasticidad. De esta manera, seremos capaces de continuar aprendiendo de manera efectiva durante toda la vida y, por ende, podremos continuar esculpiendo nuestro cerebro a través de la experiencia, pues, como aseguran los estudiosos de la materia, el aprendizaje se basa en la consolidación de sinapsis preexistentes.

Entonces, podríamos preguntarnos ¿de qué manera todos estos hallazgos de las neurociencias pueden ser utilizados por la educación para explotar el gran potencial de nuestro cerebro en favor del aprendizaje?

Como se ha mencionado previamente, al cerebro le estimulan los cambios, lo novedoso, la emoción; por tanto, es lógico concluir que ambientes de aprendizaje variados van a favorecer los aprendizajes, es decir, salidas de estudio, cambios de escenario de clase, actividades diferentes, innovación en la metodología, etc. son buenas ideas para empezar a establecer nuevas y fortalecidas redes neuronales.

Según Blakemore, y Frith, (2008) "Es un hecho científico que las áreas sensoriales del cerebro solo pueden desarrollarse cuando el entorno contiene diversos estímulos sensoriales: imágenes, texturas y sonidos; asimismo, para el desarrollo normal del cerebro son necesarias tanto la cultura como la naturaleza".
De esta manera, se encuentre ligado a nuestro curso $\mathrm{o}$ no se encuentre estrechamente relacionado, el exhortar a nuestros estudiantes para que asistan a una velada cultural, o a una exposición de arte moderno o quizás a manifestaciones artísticas propias de nuestro legado, el comentarles que tal o cual pieza teatral, conferencia o exposición de interés se están dando, es una forma de contribuir a su desarrollo y a la consolidación de nuevos aprendizajes.

Inclusive, se ha comprobado que la música modifica favorablemente nuestra estructura encefálica, en especial, las vías corticoespinales, las vías piramidales y el cuerpo calloso. Además, se ha demostrado, a través de un experimento realizado con estudiantes universitarios, que al aprender, ayudados por una serie de movimientos acompañados por música, se generaba un aumento considerable de la conectividad estructural en la vía de materia blanca que une las regiones auditiva y motora en el hemisferio derecho del encéfalo.

Según un estudio del Instituto Tecnológico de Massachusetts (MIT), la actividad cerebral registrada durante una clase expositiva es similar a la hallada cuando vemos televisión, es decir, prácticamente inexistente. Lo que sucede es que, al parecer, nuestros estudiantes se "desconectan" cuando nos escuchan disertar por largos momentos o, buscan refugio en alguna novedad de la cual puedan enterarse a través de su smartphone, lo cual es muy preocupante para cualquier docente dedicado a la enseñanza. Al parecer, estamos recorriendo caminos antiguos que antes fueron muy transitados pero que ahora no nos conducen al destino a donde deseamos dirigirnos.

Según Kolb (1984) cuando el ser humano aprende, interactúan cuatro capacidades diferentes:

Capacidad de Experiencia Concreta (EC): ser capaz de involucrarse por completo en experiencias nuevas con fines de aprendizaje

Capacidad de Observación Reflexiva (OR): En base a una experiencia concreta, el estudiante 
reflexiona sobre lo experimentado para obtener más información o profundizar su comprensión sobre el tema.

Capacidad de Conceptualización Abstracta (CA): Basado en la experiencia, el estudiante teoriza, clasifica o generaliza su experiencia, en un esfuerzo por generar nueva información.

Capacidad de Experimentación Activa (EA): Involucra el ser capaz de emplear estas teorías para tomar decisiones y solucionar problemas.

Como podemos apreciar, en cada etapa del ciclo experiencial de Kolb se produce un tipo específico de aprendizaje: el estudiante experimenta, reflexiona, piensa o hace, no permanece en ningún momento sentado, solo a la espera de que concluya el tiempo de clase.

En su libro, El arte de cambiar el cerebro, el Dr. James Zull (2014) sugirió cómo el famoso modelo de cuatro fases del ciclo de aprendizaje de David Kolb se puede mapear en cuatro grandes procesos cerebrales.

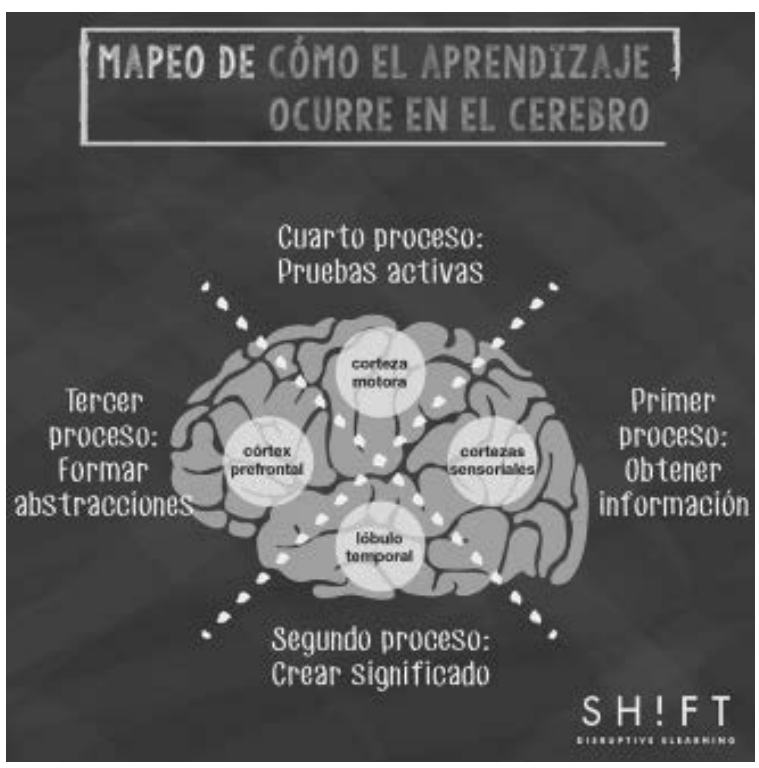

Tomado del libro El arte de cambiar el Cerebro de Dr. James Zull.

Modelo de cuatro fases de aprendizaje de David Kolb. p. 35.

Zull afirma que cada una de las fases de aprendizaje identificadas por Kolb corresponden, a su vez, a cuatro procesos que se dan en nuestro cerebro. El primero tiene que ver con la recopilación de información a través de las cortezas sensoriales. Ellas son las que registran a través de los sentidos lo que vendría a ser la "materia prima" de una experiencia nueva vivida por el aprendiz.

El segundo proceso tiene que ver con la creación de significados mediante la reflexión de lo experimentado previamente. Si somos capaces de encontrar un significado en lo que estamos aprendiendo, la información nueva va a pasar el primer filtro del cerebro, hacia la memoria de trabajo.

El hecho de proporcionar a los estudiantes preguntas de reflexión o tareas integradoras puede ayudarlos a que aumenten sus posibilidades de formular reflexiones válidas sobre el tema explorado.

El tercer proceso involucra la corteza prefrontal, el raciocinio. Es en este momento que el estudiante está listo para establecer relaciones entre la información recogida a través de la experiencia y la ya almacenada en la memoria. Igualmente, se encuentra preparado para elaborar sus propias abstracciones, fruto de los significados que construyó previamente. Es así que se genera un nuevo conocimiento en el cerebro del aprendiz.

Respecto a la generación de un nuevo conocimiento, Richland, Bjork, Finley y Linn (2005), mencionan que "...hay que fomentar la creación de conocimiento en lugar del consumo pasivo de contenido, esto dará lugar a un aprendizaje más duradero".

El cuarto proceso implica el uso de la corteza motora. Esta parte del cerebro humano convierte lo abstracto, en este caso, las ideas generadas por el aprendiz, en acciones que realizará luego de que su cerebro haya interpretado la experiencia mediante la reflexión y los significados construidos. James Zull (2014) denomina a este proceso el de las pruebas activas.

¿Cuáles pueden ser consideradas pruebas activas? 
Aquí el tema tiene que ver con que el aprendiz "haga algo", se "active"; por lo que una prueba de su actividad de aprendizaje puede ser la lectura de un material distinto, relacionado con la información recientemente aprendida.

También puede serlo el explicar o discutir lo aprendido con un compañero/a, indagar en nuevas fuentes, preguntar a personas que conozcan más sobre la temática $y$, sobre todo, poner en práctica lo aprendido. $\mathrm{Si}$ no hay trabajo aplicativo realizado, autorregulado $y$ evaluado por el propio aprendiz, es probable que no llegue a consolidarse el nuevo aprendizaje.

Todos estos aportes de la neurociencia sobre la manera cómo aprende el cerebro humano nos brindan una explicación, probablemente, todavía incompleta sobre lo que ocurre en nuestro interior cuando aprendemos. Sin embargo, y aun cuando todavía quede mucho por explorar, estos hallazgos son valiosos y nos orientan a reflexionar sobre nuevas formas de concebir la enseñanza.

Por tal motivo, y con miras a aprovechar de una mejor manera las increíbles posibilidades que poseen los cerebros de nuestros estudiantes, tendríamos que cambiar la lógica de enseñanza que hemos venido utilizando desde hace muchos años atrás.

Es necesario, por ejemplo, pasar de lo lingüístico a lo visual. Sustituir el discurso teórico oral por una interacción educativa lo más vivencial posible, apoyada por imágenes, mapas conceptuales, mapas mentales, uves heurísticas, vídeos ad hoc, visitas de estudio y demás estrategias que estimulen al estudiante a aprender. Porque es sabido que frente a estímulos novedosos se activa toda la corteza cerebral $y$, al hacerlo, se ve favorecida la integración del pensamiento, punto de partida para la construcción de nuevos aprendizajes.

Por otro lado, y tal como se ha venido desarrollando en contenidos previos, la emoción está presente de forma permanente en el devenir humano y, además de presente, sabemos que se encuentra relacionada con la cognición, y a través de ella, con el aprendizaje.
Al respecto, la neurociencia ha identificado que el camino recorrido por la nueva información pasa, primeramente, por la parte emocional del cerebro; es decir, por el sistema límbico, de allí es enviada hacia la corteza prefrontal, a fin de que se consolide en ella el aprendizaje. Por lo tanto, en este proceso se pasa de lo emocional a lo analítico.

Sin embargo, es necesario tener en cuenta que las estructuras emocionales que poseemos pueden constituirse tanto en un elemento de ayuda, como en un obstáculo para aprender.

En este sentido, afirman diversos estudiosos del cerebro, toda aquella emoción experimentada por el estudiante al momento de iniciar un aprendizaje va a afectar su desempeño. De esta manera, si el clima de la clase es positivo, va a sentirse optimista, entusiasmado, motivado con respecto al estudio y hacia la experiencia de aprendizaje que se le propone.

Por lo contrario, la crítica o palabras hirientes de un docente, en el mismo contexto, pueden generar emociones de preocupación, ansiedad o frustración, lo que podría afectar su capacidad de comprensión y análisis, pues se encontraría influenciada por la emoción que experimenta la persona.

En este contexto, según lo hallado por la neurociencia, es importante acercarnos a la parte emocional del estudiante, ayudarlo a través de la motivación y la novedad de nuestra propuesta de enseñanza, de manera que la información que deseamos sea registrada permanezca, y el aprendizaje resulte eficaz. Por ejemplo, el recurso de relatar historias como parte de la enseñanza es una manera magnífica de activar estructuras subcorticales $y$ aprender mejor.

Según Jensen (2004), citando a Le Doux (1994), las emociones dirigen la atención del ser humano, crean significados y generan sus propias vías de recuerdo. Al respecto, Jensen menciona que nuestro lado racional puede fijarse una meta de estudio o de trabajo, pero son las emociones las que nos van a dar la pasión para luchar por ese objetivo. 
Por su parte Jack Mayer (2000) señala que "las emociones transmiten tanta información, como los datos o la lógica (...) tres descubrimientos han cambiado nuestro modo de pensar sobre ellas, en primer término, el de las vías físicas y prioridades de las emociones; en segundo término, los hallazgos sobre los componentes químicos del cerebro implicados en ellas; en tercer lugar, un vínculo entre estas vías y los componentes químicos con el aprendizaje y el recuerdo cotidianos".

A su vez, Gerardo Casas Fernández (2003), menciona que "Los estudiantes que se sienten ansiosos, enfurecidos o deprimidos no aprenden; la gente que se ve atrapada en esos estados de ánimo no asimila la información de manera eficaz ni la maneja bien. Las emociones desagradables y poderosas (ira, ansiedad, tensión o tristeza) desvían la atención hacia sus propias ocupaciones interfiriendo el intento de concentración en otra cosa. Cuando las emociones entorpecen la concentración, lo que ocurre es que se paraliza la capacidad mental cognitiva que los científicos llaman "memoria activa", la capacidad de retener en la mente toda la información que atañe a la tarea que estamos realizando".

Si un estudiante se encuentra experimentando emociones desagradables va a generar un exceso de cortisol, que es una hormona asociada al estrés y, tal como señala, Gerardo Casas, serias dificultades para aprender.
Emociones, pensamientos y actitudes negativas son, además, relacionadas a enfermedades porque generan un aumento de sustancias nocivas en el torrente sanguíneo produciendo cambios en el funcionamiento del organismo y, al parecer también, la aceleración del proceso de envejecimiento.

Por el contrario, cuando la persona experimenta emociones agradables durante la sinapsis, se liberan dopamina, serotonina y endorfinas, por lo cual la transmisión del mensaje se da con mayor rapidez y facilidad; es decir se comprende y aprende con mayor eficacia.

Desde la neurociencia se recomienda, entonces, estimular el cerebro de manera positiva, a fin de que secrete dichos neurotransmisores en mayor cantidad, lo que supone un ambiente de clase grato, flexible, amable, donde haya oportunidad para desarrollar la creatividad, donde se estimule al estudiante para que genere nuevos aprendizajes.

Explorar temas nuevos mediante la indagación, trabajar colaborativamente, ponerse de pie, cambiar de aula, probar nueva metodología, hacer clases en jardines, en laboratorios, en biblioteca, estirar el cuerpo, escuchar música, etc.

Si escuchamos las recomendaciones de la neurociencia, lo que se trata es de proponer nuevas experiencias que permitan desarrollar el enorme potencial que tiene el cerebro humano, a través de lo que realicemos en el aula. 


\section{REFERENCIAS}

Diffusion tensor MRI tractography reveals increased fractional anisotropy (FA) in arcuate fasciculus following music-cued motor training. Brain and Cognition. Vol. 116, August 2017, 40-46. Recuperado de: https://doi.org/10.1016/j.bandc.2017.05.01

Gardenfors, P. (2005) La intuición como conocimiento implícito, en Revista Filosofía, Universidad Costa Rica, Vol. XLIII, N. ${ }^{\circ} 108,51-55$.

Gigerenzer, G. (1998) Visions of rationality. En Trends in Cognitive Sciences, Vol. 2, 206-214.

Kaplún, M. (2010) Una pedagogía de la comunicación, en Educomunicación: más allá del 2.0. Coordinador Roberto Aparici. Comunicación educativa.

Kearney, N. (2001) Comunidades de aprendizaje: un enfoque pedagógico de futuro. Recuperado de:http://www.camafu.org.mx/index.php/alianzas-estrategicascomunidades-y-redes-/articles/ comunidades-de-aprendizaje-un-enfoquepedagogico-de-futuro.html

Kolb A, Kolb DA. (2005) Experiential Learning Theory Bibliography: Recent Research. Recuperado de: www.learningfromexperience.com

7. Kolb A, Kolb DA. Experiential Learning Theory: A Dynamic, Holistic Approach to Management Learning, Education and Development. Weatherhead School of Management Case Western Reserve University. In: Armstrong, S. J. \& Fukami, C. (Eds.) Handbook of Management Learning, Education and Development. London: Sage Publications, 2008.

Kolb, D. (1984) Experiential Learning: Experience $k$ the Source of Learning and Development. Englewood Cliffs, NJ: Prentice Hall.

Luna, M. (2003) Itinerarios del conocimiento: formas dinámicas y contenido. Un enfoque de redes. México: Anthropos. Maclean PD. (1990) The triune brain in evolution: Role in paleocerebral functions. New York: Plenum Press.

Monroy, B, Ochoa M. (1997) ¿Pueden las redes contribuir a la modernización de la escuela? en: Francia Perspectivas. Revista Trimestral de Educación ed. Vol. 27, fasc. 295-303.

Morgane, P.J., y Mokler, D.J. (2006) The limbic system: continuing resolution. Neuroscience and Biobehavioral Reviews. Vol. 30, 119-125.

Rajmohan, V., y Mohandas, E. (2007) The limbic system. Indian Journal of Psychiatry. Vol. 49, N. ${ }^{\circ} 2,132-139$.

Roxo, M.; Franceschini, P.R.; Zubaran, C.; Kleber, F; and Sander, J. (2011) The Limbic System Conception and Its Historical Evolution. The Scientific World Journal, Vol. 11, 2427-2440.

Tversky, A. y Kahneman, D. (1981) The framing of decision and the psychology of choice. Science, Vol. 211, 453-458.

Zull, J. (2014) El arte de cambiar el Cerebro. Buenos Aires: Editorial Hobbs-Sudamericana S.A.

Fecha de recepción: 26-09-17

Fecha de aceptación: 19-10-17. 
